\section{Zinc and Copper Concentrations in Breastmilk}

The concentration of essential trace elements in breastmilk is an important topic in infant nutrition. The interesting paper by Örun, et al. [1] needs to be put in context in the light of previous research on the topic. Comprehensive reviews of breastmilk zinc [2,3] and copper [4] concentrations have addressed the maternal (constitutional and environmental) factors modulating the secretion of these essential nutrients. I was surprised that the brief discussion by Örun, et al. [1] overlooked these sources. Also, compared to international data, they showed a surprisingly low mean milk-zinc concentration.

I take the opportunity to remind readers that in the case of zinc, the stage of lactation is the only variable associated with important changes in milk concentrations. In the first days postpartum, the zinc concentrations in breast-milk fall steeply, and after the first weeks, gradually decrease throughout the following 6 weeks; reaching relatively stable levels by the third month. In spite of increasing volumes of milk consumed by nursing infants, the total zinc intake falls as a function of the rate of decrease in the milk metal concentration. For postpartum days $1,7,15,30,60,90,120$, and 180 , the reported median zinc concentrations $(\mathrm{mg} / \mathrm{L})$ are 8.12 , $4.56,3.36,2.65,1.66,1.35,1.2,0.98$ and 0.93 ; respectively [2]. Experiments with maternal zinc supplementation aimed at enriching breast-milk have not been effective. However, maternal zinc therapy has been reported to correct abnormalities of zinc metabolism during gestation and lactation [2].

The variability of breast-milk zinc concentrations is wide; it does not depend on maternal zinc intake but it can vary within and between mothers as a function of parity, stage of lactation, and type (fore or hind milk) of feeding [2]. Therefore, to test a hypothesis that a significant association exists between this variable and anthropometry (changing in the opposite direction) requires a more appropriate study design. However, it is possible that repeated measurements of both milk zinc and anthropometry in exclusively breastfed infants, as in follow-up studies, has been useful in generating the hypothesis that the decrease in zinc concentrations can be associated with weight and linear growth [5].

\section{REFERENCES}

JosÉ G DÓREA

Department of Nutrition, University of Brasilia, 70919-970 Brasília, DF, Brazil, dorea@rudah.com.br

1. Örun E, Yalçin SS, Aykut O, Orhan G, Morgil GK. Zinc and copper concentrations in breastmilk at the second month of lactation. Indian Pediatr. 2012;49:133-5.

2. Dórea JG. Is zinc a first limiting nutrient in human milk? Nutr Res. 1993;13:659-66.

3. Dórea JG. Zinc in human milk. Nutr Res. 2000;20:164587.

4. Dórea JG. Iron and copper in human milk. Nutrition. 2000;16:209-20.

5. Dórea JG. Zinc deficiency in nursing infants. J Am Coll Nutr. 2002;21:84-7.

\section{Inequity in Childhood Immunization}

Mathew, in a mammoth exercise, has looked at a large number of reports examining the coverage of routine vaccinations (RI) in various settings evaluating multiple factors to assess disparities in RI in different populations groups in India [1]. Criteria such as urban and rural communities, parental economic status, number of siblings etc have been evaluated using diverse methodology. The conclusions listed in the box bring out what is well known. Parents who are not informed of the benefits of protective immunizations do not undertake the "trouble" to get the child vaccinated or drop out without completion of the recommended doses. Similarly basic health care services, which are provided free of cost, often remain unutilized.

A recent randomized controlled study [2] carried out in low income sites in Karachi, Pakistan, including urban and peri-urban communities, examined if improving maternal knowledge of vaccination had an impact on infant immunization rates. The intervention consisted of conveying three key messages to the family explaining $(i)$ how vaccines saved children's lives, (ii) logistic information about vaccination centers, and (iii) significance of retaining immunization cards. These were communicated using easy-to-understand pictorial cards by trained child health workers (CHWs). The control group received general health promotional messages also given by CHWs. The intervention group showed an 